\title{
CYSTIC DISEASE OF THE LUNG IN THE NEWBORN TREATED BY PNEUMONECTOMY
}

\author{
BY \\ N. S. CLARK, R. C. NAIRN and F. J. SAMBROOK GOWAR \\ From the Royal Aberdeen Hospital for Sick Children
}

(RECEIVED FOR PUBLICATION MARCH 19, 1956)

Congenital cystic disease of the lung is an uncommon condition which may remain asymptomatic for long periods or may, at any time, become potentially lethal either as a result of infection or of increasing tension in the cysts. Infection is a constant danger whenever a cavity communicates with the bronchial tree and is responsible for the development of symptoms in a considerable number of cases. Ravitch and Hardy (1949) found evidence of infection in every one of 12 lung cysts excised in childhood. That infection may arise at a very early age is shown by the third case of Swan and Aragon (1954) in which a large air-containing cyst, shown radiologically at the age of 6 days, had been transformed into a tense abscess two weeks later. In early infancy, however, acute symptoms are more often produced by rising tension in the cysts and it seems that, if this complication is to develop during childhood, it nearly always does so in the first year, and, indeed, in the majority of cases, during the first few weeks of life. The early diagnosis of tension cysts has assumed increasing importance since it has been realized that surgical treatment can retrieve or prevent the desperate situation which may arise when a valvular obstruction in the bronchus causes the cysts to balloon thus increasing compression of the normal lung tissue.

The first successful resection for this condition in a young infant was reported by Fischer, Tropea and Bailey (1943). Swan and Aragon (1954) reported three successful resections for congenital cystic disease of the lung and quote references to 15 other resections in infancy after the original case of Fischer et al. In 15 of these 19 cases symptoms were due to increasing tension in the cysts. In addition we have found reports in the British and American literature of a further nine cases of tension cysts in infancy treated by resection (Walker, Taggart and Staton, 1948; Strode, Case 2, 1949; Dugan and Samson, Cases 1 and 4, 1950; Hill, 1951; Whitesell and White, 1952; Belcher and Siddons, three cases, 1954). Of this total of 24 cases, 21 were subjected to lobectomy and three to pneumonectomy with only one post-operative death (Strode, 1949) and a satisfactory functional result in all other cases.

The fact that only four of these 24 resections were performed in Great Britain (Belcher and Siddons, 1954; Crowe, 1954) has prompted us to report the following case to draw attention to the possibilities of radical surgical treatment at an extremely early age.

\section{Case Report}

Baby S.C. was the first child of healthy parents, born on November 10,1954, by low forceps delivery after an uneventful pregnancy and a labour lasting 12 hours. Her birth weight was $7 \mathrm{lb} .4 \mathrm{oz}$. and her condition at birth was satisfactory. During the third day her breathing became laboured and she was transferred to the Royal Aberdeen Hospital for Sick Children. On admission her general condition was satisfactory, she did not appear distressed despite a respiratory rate of 80 per minute and she showed no cyanosis. On examination it was apparent that the heart lay in the right chest: the left chest showed little respiratory movement and was hyper-resonant with weak breath sounds, while normal breath sounds were heard on the right side. Physical examination was otherwise negative. A radiograph of the chest (Fig. 1) showed marked displacement of the mediastinum to the right and only a small area of aerated lung was visible on that side: in the left hemithorax clearly defined cystic spaces occupied almost the whole lung field. While the appearances were suggestive of multiple tension cysts in the left lung it was felt that the possibility of diaphragmatic hernia could not be absolutely excluded and, on the following day, a barium enema, and subsequently a small 'dionosil' meal were given: the gastro-intestinal tract was shown to be normally situated.

After admission the respiratory rate fell to 50 to 60 per minute and as the infant was not distressed and was able to take her feeds no immediate treatment was considered necessary. Her condition remained unchanged until the afternoon of November 19 when she became very dyspnoeic and cyanosed. Thirty-five millilitres of air 
were aspirated through a needle in the left anterior axilla with some immediate improvement. It was impossible to withdraw more air from this site and the needle was removed. Two hours later she again became very dyspnoeic and appeared in extremis: the left chest was again aspirated, and by altering the position of the needle from time to time it was possible to remove $190 \mathrm{ml}$. of air: from no one position was it possible to aspirate more than 30 to $40 \mathrm{ml}$. which suggested that the cysts did not inter-communicate very freely. This aspiration produced considerable improvement but it was clear that any advantage gained in this way was likely to be temporary and that catheter drainage would not produce satisfactory decompression. It was, therefore, decided that immediate resection of the cystic lung was the only possible course. One further aspiration was necessary while she was being prepared for operation. A radiograph of the chest immediately before operation showed little change: if anything, the mediastinum was displaced even further to the right than in the initial radiograph.

Operation was undertaken (F.J.S.G.) on the evening of November 19, when the child was 9 days old. Premedication was with atropine, grain 1200 , and after an open ether induction, a gum elastic catheter was passed into the trachea and the lungs inflated with pure oxygen plus occasional traces of ether. The anaesthetic presented much greater difficulty than the operation: it was impossible to maintain a good colour, and inflation remained difficult after the lung was removed, presumably because of partial atelectasis of the right lung.

The left chest was opened in the fifth intercostal space with the infant in the lateral position. The lung was bulky and did not deflate normally. The upper lobe was small and atelectatic, and only a rudimentary fissure was

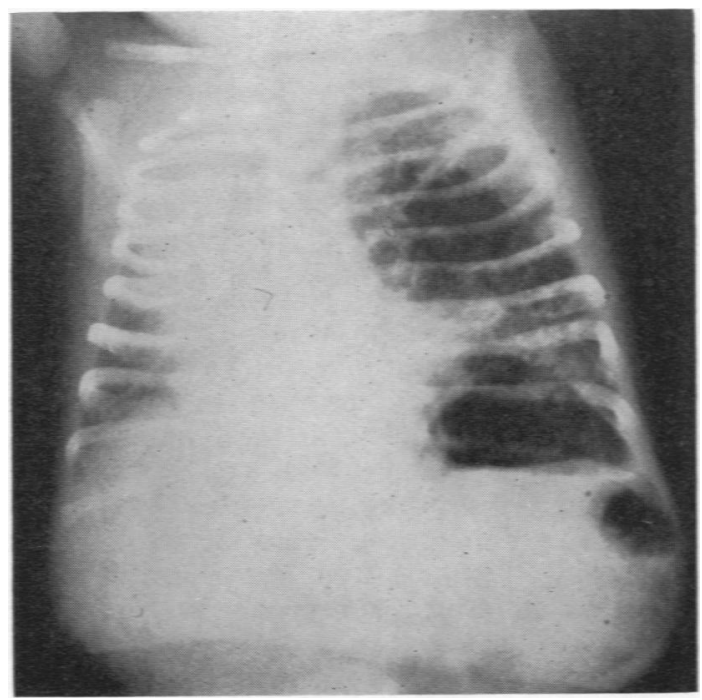

Fig. 1.-Age 3 days. Gross mediastinal displacement by air-distended cysts in the left lung. present. As speed was essential, no attempt was made to preserve the upper lobe, and dissection pneumonectomy was carried out. The bronchus stump was closed with interrupted nylon sutures and covered with pleura. The chest was closed in layers without drainage, the pleural pressure being adjusted by means of a needle to a mean minus $5 \mathrm{~cm}$. of water after the infant had been turned on her back.

On return from the theatre her general condition was poor and she required continuous oxygen. Twelve hours later she was crying quite lustily and her respirations were much easier though her colour still deteriorated when oxygen was withheld. A radiograph showed comparatively little expansion of the right lung. The pressure in the left pleural cavity was found to be -2 to $-18 \mathrm{~cm}$. of water: $260 \mathrm{ml}$. of air was aspirated, leaving a final pressure of -11 to $0 \mathrm{~cm}$. water. Further aspirations of $80 \mathrm{ml}$. that evening and $20 \mathrm{ml}$. next morning (November 21) maintained a satisfactory negative pressure and by November 22 radiography showed the right lung well expanded and apparently normal and the mediastinum well over into the left chest, which now contained an effusion. It was now possible to stop the administration of oxygen and her respirations remained easy and her colour good. Twenty-five millilitres of blood-stained fluid were aspirated from the left chest on November 27, but thereafter no further aspiration was necessary. Radiographs taken at intervals until her discharge from hospital on December 18 showed further shift of the mediastinum to the left and considerable elevation of the left diaphragm. She remained rather hypotonic and easily tired, and catheter feeding was necessary until a few days before her discharge: her general condition then improved and she began to take her feeds by bottle.

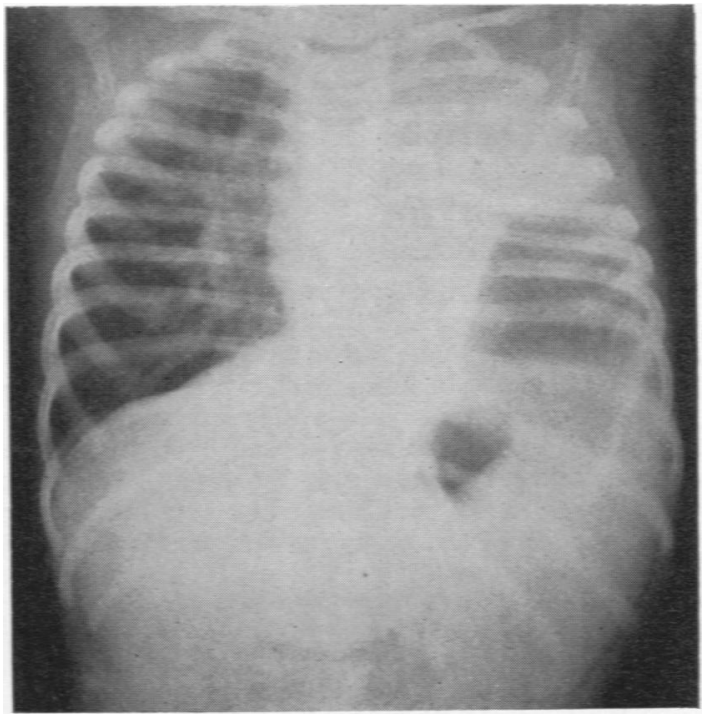

Fig. 2.-One year after operation. The left diaphragm has risen considerably and the mediastinum has moved further to the left. 
Her progress at home, although slow at first, was steady, and by the age of 4 months she weighed $12 \frac{1}{4} \mathrm{lb}$., looked well and held her head up steadily. At the age of 1 year she weighed $20 \frac{1}{4} \mathrm{lb}$. and looked a normal child with a remarkably symmetrical chest and no sign of scoliosis. She could stand with minimal support, was not dyspnoeic and had thrown off several respiratory infections without difficulty. A radiograph of the chest (Fig. 2) showed considerable displacement of the mediastinum to the left and marked elevation of the left cupola of the diaphragm: the right lung appeared normal.

Pathology. The resected lung was fixed before examination by introducing formol-acetate into the bronchial tree under slight positive pressure and tying off the main bronchus. Transection showed diffuse cystic change in the lower lobe except for a small area in the apical segment which was atelectatic (Fig. 3). The upper lobe was poorly developed and not well demarcated from the rest of the specimen; it had a similar appearance to the apical segment of the lower lobe. The cysts in the lower lobe varied in diameter from the lower limit of visibility up to $3 \mathrm{~cm}$.: the lining of the larger ones was often trabeculated as though they had been formed by the confluence of several smaller cysts.

Microscopical examination showed that the cystic spaces were bronchiectatic in nature. They were lined mainly by ciliated columnar epithelium which was pseudostratified in some places: occasionally the epithelium was cuboidal and it was more or less deficient where the cysts opened into alveolar ducts. Normallooking alveoli were found between the cystic spaces (Fig. 4). The atelectasis in the airless upper zones was confirmed: the lung tissue here showed foci of maldevelopment in which the parenchyma appeared to be of foetal type and there were also areas of alveolar haemorrhage.

In view of the special interest of the case a more detailed histological examination of the lung was made to try to decide the pathogenesis of the cysts and also the cause of their valvular inflation. Large serial sections, $25 \%$ thick, were cut from the whole of the upper half of the specimen illustrated in Fig. 3. Two hundred and fifty of these sections were stained alternately with haematoxylin and eosin and Verhoeff's elastic stain counterstained by van Gieson's method. Extra sections from here and elsewhere in the lung were stained for special features by Foote's silver method, by the per-iodicacid-Schiff method, and by Gram's method. The following observations were made from study of these sections. Most of the cysts looked like dilated bronchioles in that they showed no cartilage in their walls: some appeared to be dilated respiratory bronchioles since they were lined partly by cubical epithelium and opened directly into alveolar ducts. The cyst walls were often lax and showed many convolutions and papillary foldings. Deep to the epithelium the wall consisted of fibrous connective tissue with abundant elastic: smooth muscle was recognized but was inconspicuous. The lumina of the cysts were part of the air conducting system of the lung, communicating proximally with the main bronchi and peripherally with alveolar ducts, alveolar sacs and alveoli. The alveolar ducts were sometimes distended, but the air sacs themselves, although showing more variation in size than usual, were in no respect cystic: for the most part they looked normal. A few of the alveoli and cystic spaces contained fresh blood, possibly from the operation or the pre-operative chest punctures. A further notable feature was the presence of broad trabeculae of loose connective tissue, dividing the lung into large lobules. There was no positive evidence of prenatal aspiration of amniotic fluid: typical squames could not be identified either in haematoxylin-and-eosin preparations or in the Gramstained sections. The main vessels and bronchi looked normal apart from certain striking changes near the origins of the segmental bronchial branches: here there was definite evidence of bronchial stenosis. A model made from tracings of photographic magnifications of this area confirmed the presence of a severe stenosis; it was a flattening rather than a uniform constriction and in some ways suggestive of a twist rather than of a compression (Fig. 5). Such a degree of stenosis could readily act as a check valve (Jackson and Jackson, 1952) and cause progressive inflation of the lung beyond.

\section{Discussion}

Pathogenesis. The classification and development of cystic changes in the lung have been discussed in detail by several authors (Koontz, 1925; Sellors, 1938; Kjaergaard, 1935; Willis and Almeyda, 1943), but perhaps the simplest concept of the mechanisms producing pulmonary cysts in the newborn is that of Anspach and Wolman (1933) who suggested that the cysts may be $(a)$ preformed but collapsed, expanding with the first respiration: $(b)$ small congenital bronchiectatic dilatations which expand with respiration if there is a concomitant check-valve deformity in the air passages; (c) fluid cysts which become air-filled after birth once the fluid is evacuated. It is to category $(b)$ that our case seems to belong, since it shows undoubted bronchiolectasis together with check-valve deformities in the bronchi. An element of bronchiolectasis appears to have been present for some time before birth because the walls of the cystic cavities were in many places lax and thrown into folds indicating the growth of extra tissue here. Such an increase in bronchiolar dimensions could hardly have arisen in the few days of post-natal life simply from the effect of the valvular anomaly exposing normal-sized bronchioles to increasing air tension. There is also indirect evidence for this view from the observations of Robertson and James (1951) and Weisel (1955) that valvular bronchial stenosis alone is associated with diffuse lobar emphysema, not dilatation of bronchi or bronchioles. Thus it is clear that, while the valvular bronchial stenosis in our case must have 


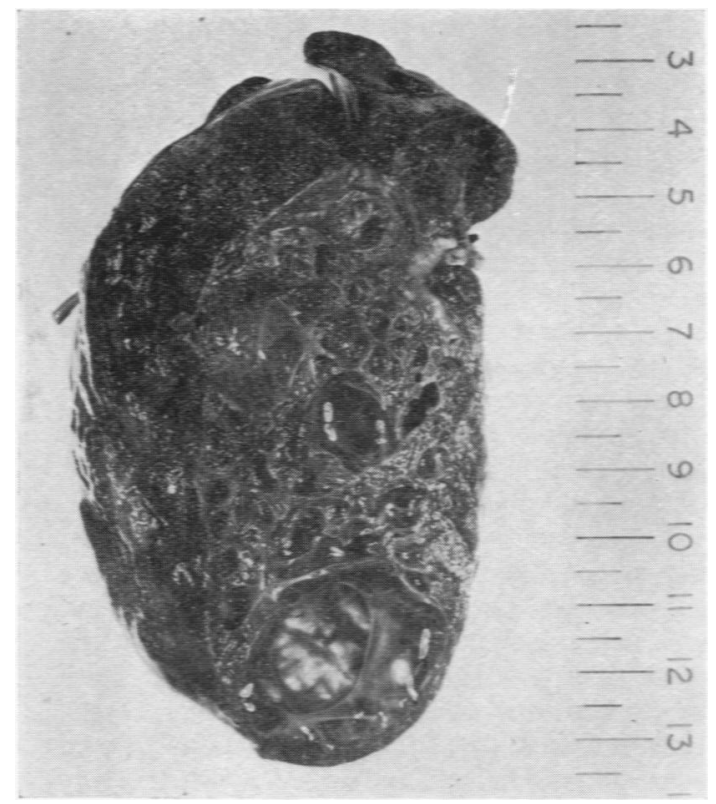

Fig. 3.-Lung transected coronally to show multiple cysts in the lower lobe; the apical segment of the lower lobe and the entire upper lobe are maldeveloped and atelectatic. (Two-thirds size.)

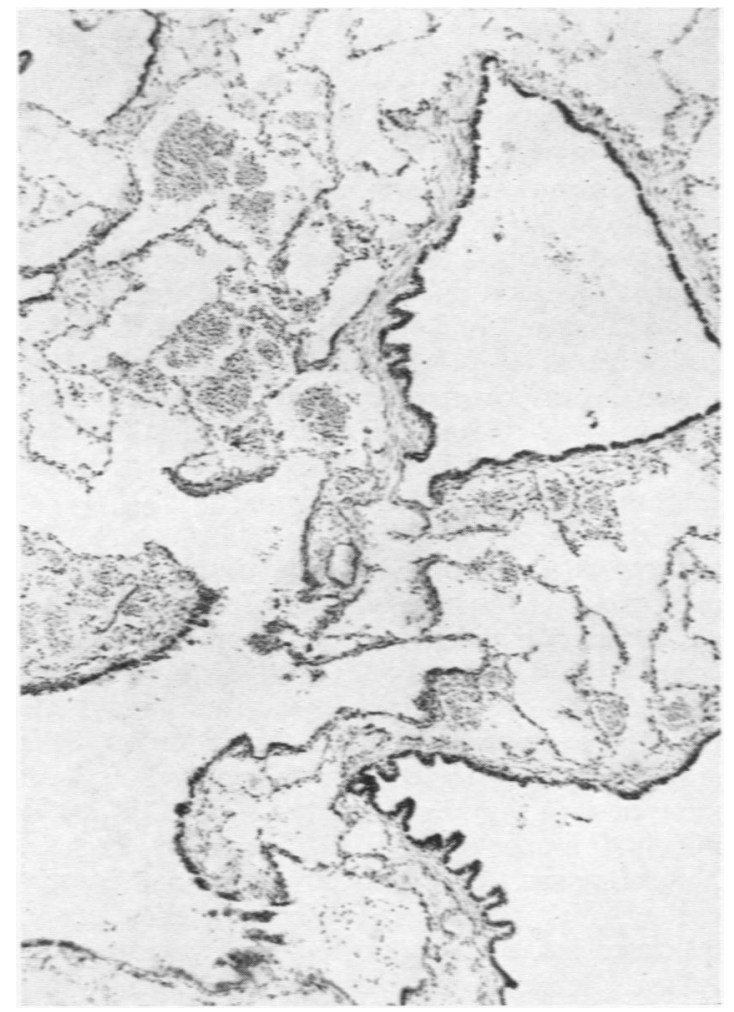

FK. 4.-Section of the lower lobe showing cysts lined by respiratory epithelium and opening into alveolar ducts; the alveoli between the cysts contain some fresh blood but look otherwise normal (Haematoxylin and eosin. $\times 60$.)

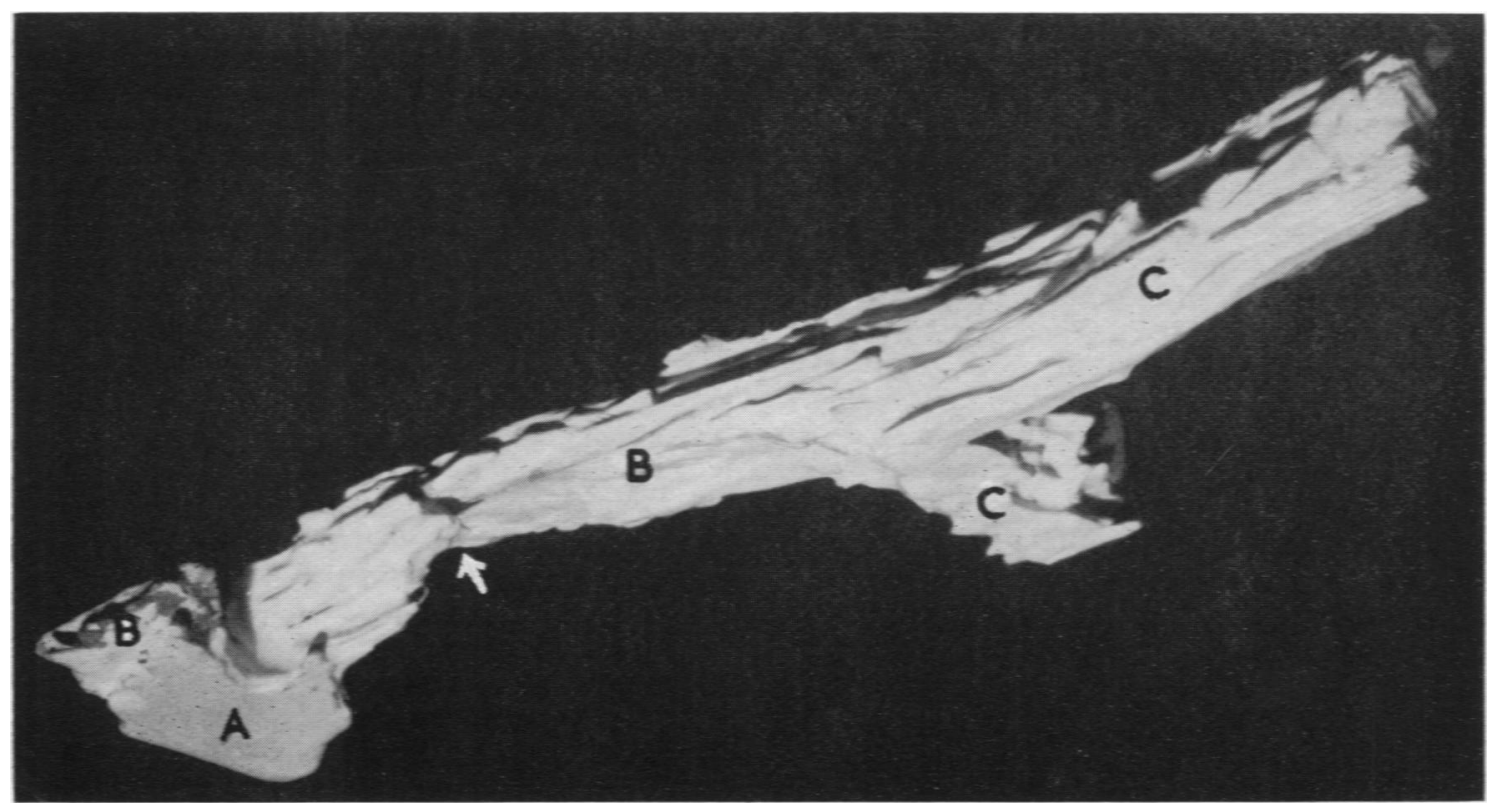

Fig. 5.- Model constructed from tracings of photographic enlargements of the serial sections from the posterior besal sezmental bronchus region. The basal bronchus (A) is seen branching into its anterior and posterior branches (B). The latter shows the stenosis marked by the arrow, and gives rise to two subsidiary branches $(C)$. $(\times 20$.) 
been of prime importance in the production of the increasing intrathoracic tension after birth, it probably played no part in the production of the weakening and overgrowth of the bronchiolar walls. In theory, it might be supposed that such a stenosis could lead to cystic lung changes in utero from the progressive aspiration of amniotic fluid, but, in fact, this is a most unlikely explanation since intrauterine breathing in human infants is no more than a hypothetical occurrence; moreover, in our case there was no evidence of aspiration histologically and no sign of residual fluid in the cysts when the child was radiographed at 3 days old.

It seems likely that the bronchial anomaly, the bronchiolectasis and the partial dysplasia of the upper zones of the lung were all parts of a general, but unilateral, pulmonary developmental disturbance. The timing of this maldevelopment can now only be a matter for speculation but if the bronchial anomaly is in fact a torsion defect, as is hinted by its appearance, then this is likely to have occurred at a very early stage of foetal life while bronchial branching was still minimal. Perhaps a concomitant local anomaly of blood supply resulted in the defective development elsewhere in the lung; but evidence for such a speculation is lacking. It seems highly probable, however, that the changes in the cystic lung had a local cause because the opposite lung was not similarly affected.

Diagnosis. Although this condition is uncommon its diagnosis should rarely present difficulty. The essential clinical features are dyspnoea in an infant showing impaired respiratory movement, distension, hyper-resonance and poor air entry over one side of the chest with gross displacement of the mediastinum into the opposite side of the chest. Radiographs may show a single 'balloon' cyst or, as in the present case, multiple cystic spaces occupying most of the affected side of the chest.

Pneumothorax, congenital lobar emphysema and diaphragmatic hernia are the only other abnormalities which can produce rather similar clinical or radiographic pictures. Pneumothorax and congenital lobar emphysema produce a clinical picture indistinguishable from that of tension cysts but the radiographic appearances are usually diagnostic. In diaphragmatic hernia the percussion note on the affected side will usually be impaired rather than hyper-resonant but the physical signs may be otherwise identical. Multiple cysts, as in the present case, may produce a radiographic picture resembling that of the bowel gas shadows in diaphragmatic hernia but the distinction can easily be made with the aid of an opaque meal or enema. Failure to carry out this investigation resulted in laparotomy in two of the 24 cases quoted in the introductory paragraphs before the correct diagnosis was reached.

Management. Caffey (1953) maintains that lung cysts in infancy usually regress spontaneously and should always be treated expectantly except in 'the rare case of acute respiratory obstruction due to sudden and marked expansion of the cysts. It is clear, however, that in nearly all the cases described in his paper the cysts followed pneumonia. Cysts of this type are by no means uncommon and it is generally agreed that they are usually benign and tend to disappear spontaneously. Caffey appears to believe that there is no fundamental difference between the two types of cyst, but we have found no convincing evidence that cysts found in the first week or two of life in the absence of infection behave in the same way as post-pneumonic cysts. It is certain that some of them will become infected and that some will become ballooned. Whether any of them disappear or remain benign and symptomless throughout life we do not know, but it seems highly probable that, if they escape the danger of over-inflation, the ultimate risk of infection must be very high. Perrott (1956) has described 17 cases of cystic disease of the lung, 15 of them aged 20 years or more; virtually all of them had signs or symptoms of infection and the majority were severely disabled. Until we know more of the natural history of these cysts it is impossible to say whether resection is justified in every case even in the absence of acute symptoms. It seems probable, however, that in the present case which showed gross mediastinal displacement, operation should have been performed earlier despite the fact that signs of respiratory distress became less severe after the baby's admission to hospital. The operation might well have been less hazardous had it been performed immediately the diagnosis was confirmed; the temptation to temporize while the symptoms seemed less acute should have been resisted and surgical advice sought at once.

Evidence of progressive compression of functioning lung by distension of congenital cysts is an absolute indication for immediate resection of the affected lung or lobe. Thoracotomy and lung resection are tolerated amazingly well by very young infants, and present no serious technical difficulties. Delay is likely to increase the dangers of operation owing to the anaesthetic difficulties which result from compression of normal lung tissue. In the present case we did not know whether the right lung was structurally normal as it was almost completely collapsed when the first radiograph was taken, but it appeared 
that left pneumonectomy offered the only hope of saving the child's life. Resection is the only measure which will at the same time relieve the immediate danger and offer prospect of permanent cure. Aspiration of the cysts is a useful expedient while preparations are made for operation, but, as Albert and Potts (1953) point out, it is never an elective procedure and is always followed by re-inflation of the cysts and often by pneumothorax. They also emphasize that when a definitive diagnosis of expanding lung cyst has been made and confirmed by roentgenographic examination, the infant is literally followed by a physician with a large syringe and needle in his hands while preparations are being made for surgery'. We had not read this paper before dealing with the present case, but were rapidly forced to the same conclusion and the precaution they suggest was in fact taken, almost to the letter.

\section{Summary}

An infant with congenital cystic disease of the lung developed acute respiratory distress at the age of 3 days as a result of progressive distension of the cysts. Symptoms became much more severe at the age of 9 days and pneumonectomy was performed with an excellent functional result. Although lobectomy has been performed for this condition at an even more tender age, it is believed that the present patient is the youngest to undergo pneumonectomy.

Examination of the resected lung showed the cysts to be dilated bronchioles which in some cases opened directly through alveolar ducts into alveoli. The evidence suggested that some bronchiolar dilatation was present before birth. The segmental bronchi serving the affected areas of the lung showed localized stenoses which presumably acted as check-valves with consequent ballooning of the cysts.

Attention is drawn to the importance of the early recognition of expanding lung cysts in infancy, a condition which, if untreated, is likely to prove rapidly fatal. A survey of the literature has shown that the results of early surgical resection are highly satisfactory.

Our thanks are due to Professor John Craig and Professor J. S. Young for helpful advice and criticism, and to Mr. R. G. M. Drummond for the illustrations.

\section{REFERENCES}

Albert, H. M. and Potts, W. J. (1953). Pediatrics, 12, 283.

Anspach. W. E. and Wolman. I. J. (1933). Surg. Gynec. Obstet., 56, 635 .

Bekher, J. R. and Siddons, A. H. M. (1954). Thorax, 9, 38.

Caffey, J. (1953). Pediatrics, 11, 48.

Crowe, G. G. (1954). J. thorac. Surg., 27, 399

Dugan, D. J. and Samson, P. C. (1950). Ibid.. 20, 729

Fischer, C. C., Tropea, F. and Bailey, C. P. (1943). J. Pediat., 23, 219.

Hill, L. F. (1951). Ibid., 38, 511.

Jackson, C. and Jackson, C. L. (1952). In Brennemann's Practice of Pediatrics, vol. 2, ch. 50, p. 2. Hagerstown.

Kjaergaard, H. (1935). Acta med. scand., 86, 407.

Koontz, A. R. (1925). Bull. Johns Hopk. Hosp., 37, 340.

Perrott, E. A. (1956). Brit. med. J., 1, 436.

Ravitch, M. M. and Hardy, J. B. (1949). Arch. Surg. (Chicago), 59, 1. Robertson, R. and James, E. S. (1951). Pediatrics, 8, 795

Sellors, T. H. (1938). Tubercle (Lond.), 20, 49.

Strode, J. E. (1949). J. thorac. Surg., 18, 404.

Swan, H. and Aragon, G. E. (1954). Pediatrics, 14, 651

Walker, J. M., Taggart, W. B. and Staton, H. J. (1948). J. Pediat., 33, 601 .

Weisel, W. (1955). Ann. Surg., 142, 17

Whitesell, F. B. and White, W. J. (1952). Ibid., 136, 299.

Willis, F. E. S. and Almeyda, J. (1943). Tubercle (Lond.), 24, 27 and 43. 